\title{
Late gadolinium enhancement cardiovascular magnetic resonance for sudden cardiac death risk stratification in hypertrophic cardiomyopathy
}

\author{
Tevfik F Ismail ${ }^{1 *}$, Andrew Jabbour ${ }^{1}$, Ankur Gulati ${ }^{1}$, Amy Mallorie', Sadaf Raza', Thomas E Cowling ${ }^{1}$, Bibek Das ${ }^{1}$, \\ Jahanzaib Khwaja', Rick Wage ${ }^{1}$, James Moon², Amanda Varnava ${ }^{3}$, Carl Shakespeare ${ }^{4}$, Perry Elliott ${ }^{2}$, Rory OHanlon', \\ Dudley J Pennell', Sanjay K Prasad ${ }^{1}$
}

From 16th Annual SCMR Scientific Sessions

San Francisco, CA, USA. 31 January - 3 February 2013

\section{Background}

Although myocardial fibrosis identified by late gadolinium enhancement (LGE) cardiovascular magnetic resonance (CMR) may predict adverse outcomes among patients with hypertrophic cardiomyopathy (HCM), its precise role in risk stratification for sudden cardiac death (SCD) remains unresolved. Previous studies have relied on broad surrogate composite endpoints and were underpowered to assess SCD risk or to adjust for confounding variables. To address this, we studied the prognostic significance of LGE in a large HCM cohort with long-term follow-up.

\section{Methods}

Consecutive patients with $\mathrm{HCM}(\mathrm{n}=711$, median age 56.3 years, interquartile range [IQR] 46.7 to $66.6 ; 70.0 \%$ male) underwent LGE-CMR and were prospectively followed for a median of 3.5 years. This amounted to a total of 2852 patient-years of follow-up. The primary endpoint was SCD or aborted SCD. The principal secondary endpoint was a composite of cardiovascular mortality and aborted SCD. LGE imaging was performed in two orthogonal phase-encoding directions $\sim 10 \mathrm{~min}$ after $0.1 \mathrm{mmol} /$ $\mathrm{kg}$ gadolinium using an inversion recovery-prepared gradient echo sequence. The amount was quantified using the full-width at half-maximum method.

\section{Results}

Patients with LGE had more significant left ventricular hypertrophy and lower left ventricular ejection fraction

${ }^{1}$ CMR Unit \& NHLI Imperial College London, Royal Brompton Hospital \& NHLI Imperial College London, London, UK

Full list of author information is available at the end of the article
(LV-EF) than those without LGE (Table 1). The median amount of LGE in the $471(66.2 \%)$ patients with enhancement was $5.9 \%$ of left ventricular mass (IQR: 2.2 to 13.3). At the end of follow-up, 22 patients (3.1\%) reached the primary endpoint: $18(3.8 \%)$ in the LGE group and $4(1.7 \%)$ in the no LGE group. The amount but not the presence of fibrosis was a significant univariate predictor of SCD risk (HR per 5\% LGE: 1.24, 95\% CI 1.06 to $1.45 ; \mathrm{p}=0.007$ and HR for LGE: $2.69,95 \%$ CI 0.91 to $7.97 ; \mathrm{p}=0.073$ respectively). However, on multivariate analysis, only left ventricular ejection fraction (LV-EF) remained significant (HR: $0.92,95 \%$ CI 0.89 to 0.95 ; $\mathrm{p}<0.001$ )[Figure 1]. For the secondary composite endpoint of cardiovascular mortality and aborted SCD, 30 (6.4\%) patients in the LGE group versus 8 (3.3\%) in the no LGE group reached the endpoint (HR for LGE: 2.24, 95\% CI 1.03 to $4.89 ; \mathrm{p}=0.043$ ). However, on multivariate analysis, only LV-EF and non-sustained VT (NSVT) emerged as a significant predictors of outcome (HR for LV-EF: $0.92,95 \%$ CI 0.90 to $0.95, \mathrm{P}<0.001$; and HR for NSVT: $3.15,1.37$ to $7.28, \mathrm{p}=0.007$ ).

\section{Conclusions}

Neither the presence nor the amount of LGE independently predicted SCD risk after adjusting for confounders. In contrast, LV-EF was the best independent predictor of SCD and cardiovascular mortality, and should therefore be considered as part of the routine risk stratification of patients with HCM.

\section{Funding}

This work is supported by the NIHR Cardiovascular Biomedical Research Unit at the Royal Brompton and 
Table 1

\begin{tabular}{|c|c|c|c|c|}
\hline CMR parameters & No LGE $(n=240[33.8 \%])$ & LGE $(n=471[66.2 \%)$ & All patients $(n=711)$ & $P$ value \\
\hline LV-EDV index $-\mathrm{ml} / \mathrm{m}^{2}$ & $67.8 \pm 14.5$ & $69.8 \pm 16.2$ & $69.1 \pm 15.7$ & 0.123 \\
\hline LV-ESV index $-\mathrm{ml} / \mathrm{m}^{2}$ & $15.9 \pm 7.5$ & $19.3 \pm 10.3$ & $18.2 \pm 9.6$ & $<0.001$ \\
\hline LV ejection fraction - \% & $77.1 \pm 7.3$ & $73.3 \pm 9.5$ & $74.6 \pm 9.0$ & $<0.001$ \\
\hline$<50 \%$ & $1(0.4 \%)$ & $11(2.3 \%)$ & $12(1.7 \%)$ & 0.021 \\
\hline $50-59 \%$ & $5(2.1 \%)$ & $25(5.3 \%)$ & $30(4.2 \%)$ & \\
\hline$\geq 60 \%$ & $232(97.5)$ & $434(92.3)$ & $666(94.1 \%)$ & \\
\hline LV mass index $-\mathrm{g} / \mathrm{m}^{2}$ & $88.7 \pm 25.1$ & $108.4 \pm 40.1$ & $101.9 \pm 37.0$ & $<0.001$ \\
\hline Maximum end-diastolic wall thickness - $\mathrm{mm}$ & $16.6 \pm 3.7$ & $20.8 \pm 5.2$ & $19.4 \pm 5.1$ & $<0.001$ \\
\hline
\end{tabular}

LV=Left Ventricular; LV-EDV=Left ventricular End-Diastolic Volume; LV-ESV=Left Ventricular End-Systolic Volume.

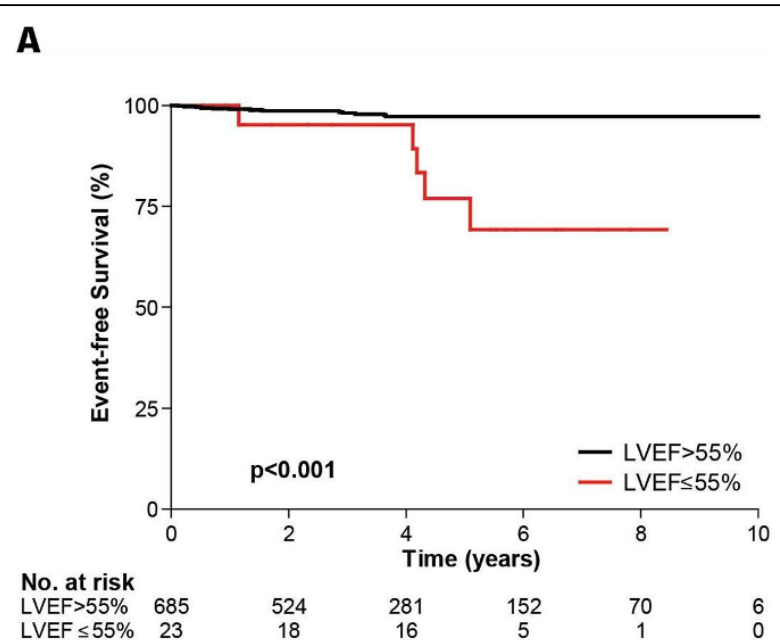

B

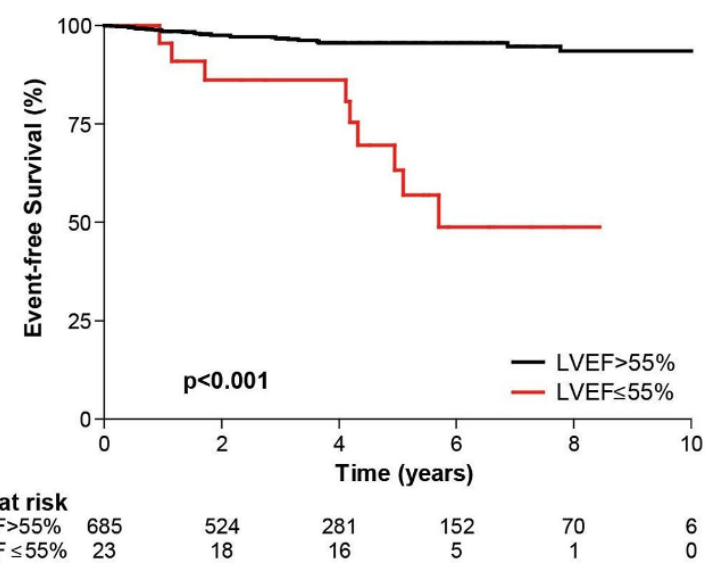

\section{Author details}

${ }^{1}$ CMR Unit \& NHLI Imperial College London, Royal Brompton Hospital \& NHLI Imperial College London, London, UK. ${ }^{2}$ Institute of Cardiovascular Science, Heart Hospital \& University College London, London, UK. ${ }^{3}$ Cardiology, West Hertfordshire Hospitals NHS Trust, Watford, UK. ${ }^{4}$ Cardiology, South London Hospitals NHS Trust, London, UK.

Published: 30 January 2013

doi:10.1186/1532-429X-15-S1-067

Cite this article as: Ismail et al:: Late gadolinium enhancement cardiovascular magnetic resonance for sudden cardiac death risk stratification in hypertrophic cardiomyopathy. Journal of Cardiovascular Magnetic Resonance 2013 15(Suppl 1):O67.

\section{Submit your next manuscript to BioMed Central} and take full advantage of:

- Convenient online submission

- Thorough peer review

- No space constraints or color figure charges

- Immediate publication on acceptance

- Inclusion in PubMed, CAS, Scopus and Google Scholar

- Research which is freely available for redistribution

Submit your manuscript at www.biomedcentral.com/submit 